\title{
Inflammatory Biomarkers in induced Sputum Predict Chronic Obstructive Pulmonary Disease (COPD) Severity
}

\section{Yuting He}

Zhongshan Hospital Fudan University

\section{Zhi Deng}

Zhongshan Hospital Fudan University

Jianjun Jin

Zhongshan Hospital Fudan University

\section{Ruolin Mao}

Zhongshan Hospital Fudan University

\section{Lei Gao}

Zhongshan Hospital Fudan University

\section{Yi Zhang}

Air Liquide Holding Co,;Ltd

\section{Gang Wang}

Sichuan University West China Hospital

\section{Peng Gao}

The Second Affiliated Hospital of Jilin University

\section{Min Xie}

Tongji Hospital of Tongji Medical College of Huazhong University of Science and Technology Huayin Li

Zhongshan Hospital Fudan University

Zhihong Chen ( $\square$ chen.zhihong@zs-hospital.sh.cn )

Zhongshan Hospital Fudan University https://orcid.org/0000-0001-6048-5917

\section{Research}

Keywords: COPD, induced sputum, airway inflammation, biomarkers, lung function

Posted Date: November 20th, 2020

DOI: https://doi.org/10.21203/rs.3.rs-111056/v1 
License: (c) (i) This work is licensed under a Creative Commons Attribution 4.0 International License. Read Full License 


\section{Abstract}

Background: The progression of an abnormal inflammatory response plays a crucial role in the functional decline that occurs in COPD patients[1].Compared to serum biomarkers, inflammatory biomarkers in induced sputum would be a more reliable reflection of the inflammatory processes in the airways. Based on this, we explored the association of inflammatory biomarkers in induced sputum with lung function in COPD patients.

Methods: 102 patients with COPD were recruited and underwent spirometry. According to the percentage of the predicted forced expiratory volume in 1 second value (FEV1\%pred), participants with COPD were divided into a mild and moderate group (FEV1\%pred $\geq 50 \%, \mathrm{n}=57$ ) and a severe and extremely severe group ( $\mathrm{FEV} 1 \%$ pred $<50 \%, \mathrm{n}=45$ ). Then we analyzed a series of inflammatory biomarkers in induced sputum and determined their association with lung function in COPD patients.

Results: We found an increased levels of eosinophils percentage, MMP9 mRNA, LTB4R mRNA, and decreased levels of CC16 mRNA and miR-155 in the induced sputum of patients with severe and extremely severe COPD. In our analysis, sputum cell CC16 mRNA expression, but not CC16 secretion in the supernatant of the sputum, was closely and independently correlated with lung function decline in patients with COPD.

Conclusion: The levels of numerous inflammatory biomarkers in induced sputum were associated with

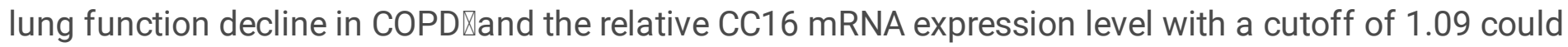
be a predictive biomarker of the severity of COPD.

\section{Introduction}

Chronic obstructive pulmonary disease (COPD) is an inflammatory airway disease characterized by progressive and persistent airflow limitation[2]. As one of the leading causes of mortality and morbidity worldwide, COPD imposes serious economic and social burdens[3]. Lung function is often assessed by measuring the forced expiratory volume in 1 second (FEV1) and the forced vital capacity (FVC)[4]. Indeed, according to the current Global Initiative for Chronic Obstructive Lung Disease (GOLD) standards, the severity and progression of COPD are graded based on the percentage of the predicted FEV1 value (FEV1\%pred)[1]. However, COPD is a complex disease with multiple phenotypes that cannot be identified through the measurement of lung function alone. The importance of COPD risk assessment, phenotype identification, and the diagnosis of exacerbation make it even more important to identify robust biomarkers for COPD.

Abnormal inflammatory responses in the lungs are usually caused by noxious particles or gases, leading to the progressive destruction of the structure and function of the lungs[5]. Inflammatory cells, such as neutrophils and eosinophils accumulate in airways, inducing the production and activation of a variety of inflammatory cytokines and proteases that participate in the chronic inflammation that is characteristic of COPD[5-7]. Previous studies have shown that tumor necrosis factor a (TNF-a), interleukin-1 $\beta$ (IL-1 $\beta)$, 
interleukin-6 (IL-6), interleukin-8 (IL-8), and leukotriene B4 (LTB4) are crucial cytokines involved in the airway inflammatory response[8-10]. Monocyte chemotactic protein-1 (MCP-1) aggregates macrophages at the site of inflammation, and matrix metalloproteinase 9 (MMP-9) mediates the accumulation and infiltration of inflammatory cells in the lung[11]. Moreover, serum surfactant protein-D (SP-D) and Clara cell 16 (CC16), which are derived from lung parenchymal cells, have been found to be associated with airflow limitation in COPD $[12,13]$. Recently, the dysregulation of microRNAs (miRNAs), such as microRNA-155 (miR-155) and microRNA-21 (miR-21), was shown to be involved in the inflammatory response or fibrosis in pulmonary diseases[14]. Systemic inflammation markers can be assessed in the peripheral blood in COPD patients; however, this method does not seem to be a sufficiently accurate method of reflecting the inflammatory processes within the airways[6, 15]. Thus, a reliable measurement of local airway inflammation in COPD should be based on samples obtained from the location of sustained inflammation. Inducing sputum has been widely acknowledged as a noninvasive and repeatable sampling method to evaluate the patterns of inflammatory cells and the concentrations of various inflammatory mediators[16].

Based on previous studies, we performed this study to investigate the relationships between lung function decline and multiple inflammatory biomarkers in the induced sputum of patients with COPD.

\section{Methods}

\subsection{Study population}

The study was approved by the Hospital Medical Ethics Committee. A total of 102 patients were recruited from Zhongshan Hospital of Fudan University (a tertiary teaching hospital) from 2017 to 2018 (Fig. 1). The diagnosis of COPD was made according to the GOLD criteria. Briefly, COPD was diagnosed when the ratio of the postbronchodilator forced expiratory volume in 1 second to the forced vital capacity (FEV1/FVC) was $<70 \%$. According to FEV1\%pred, participants with COPD were divided into a mild and moderate group ( $F E V 1 \%$ pred $\geq 50 \%, n=57$ ) and a severe and extremely severe group (FEV1\%pred $<50 \%$, $n=45$ ). In addition to the GOLD criteria, we also adopted the St George's Respiratory Questionnaire (SGRQ), modified Medical Research Council (mMRC) scale and COPD Assessment Test (CAT) to evaluate the progression of COPD. For all participants, baseline characteristics were recorded, including age, sex, symptoms, complications and treatments. In addition, participants who had met any of the following criteria in the previous year were recorded as having experienced acute exacerbations: 1) hospitalization $\geq$ once per year or 2) ambulatory treatment $\geq$ twice a year.

\subsection{Spirometry}

Spirometry was performed by a trained respiratory technician according to the American Thoracic Society recommendations. FVC and FEV1 were measured, and the FEV1\%pred and FEV1\%FVC were calculated.

\subsection{Fraction exhaled nitric oxide (FeNO)}


Multiflow FeNO was evaluated with a NO analyzer (FeNo Expair, Medisoft, Sorinnes - Belgium). In the seated position, patients were asked to exhale all the air from their lungs and then to inhale as deeply as possible for 5 seconds. Then, they exhaled slowly for 10 seconds. The flow rate was maintained at $50 \mathrm{~mL} / \mathrm{s}$ during the detection period. The FeNO test was repeated 3 times, and the mean value was calculated. This process was performed in strict accordance with the operating instructions.

\subsection{Sputum induction}

Patients with COPD inhaled $200 \mathrm{\mu g}$ salbutamol, and then the production of sputum was induced by the inhalation of $4.5 \% \mathrm{NaCl}$ for $5 \mathrm{~min}$. All sputum samples were processed immediately. A $0.1 \%$ solution of dithiothreitol was added to the sputum and shaken for $10 \mathrm{~min}$. Then, a double volume of phosphatebuffered saline (PBS) was added, and the mixture was vortexed briefly. After filtration through two layers of sterile gauze, the sputum was centrifuged for $10 \mathrm{~min}$ at $1800 \mathrm{~g}$. After centrifugation, the supernatant and sediment were collected and stored at $-80^{\circ} \mathrm{C}$ until biomarker analysis.

\subsection{Induced sputum cell count}

The cytospin slides were stained with May-Grunwald-Giemsa stain to determine the differential cell count, and a slide with $\geq 400$ cells was identified as an acceptable sample. The induced sputum was considered acceptable if it contained less than $20 \%$ squamous epithelial cells. Based on the percentages of neutrophils and eosinophils, the inflammation in the induced sputum sample was classified as one of four phenotypes, namely, the neutrophil phenotype, eosinophil phenotype, mixed granulocyte phenotype and paucigranulocytic phenotype. The neutrophilic phenotype was defined as a sample with $\geq 61 \%$ neutrophils and $<3 \%$ eosinophils; the eosinophilic phenotype was defined as a sample with $\geq 3 \%$ eosinophils and $<61 \%$ neutrophils; the mixed phenotype was defined as a sample with $\geq 61 \%$ neutrophils and $\geq 3 \%$ eosinophils; and the paucigranulocytic phenotype was defined as a sample with $<60 \%$ neutrophils and $<3 \%$ eosinophils[17].

\subsection{Total RNA extraction and qRT-PCR}

Based on previous studies, we evaluated the association of lung function with the following inflammatory biomarkers: A1 adenosine receptor (A1AR), CC16, interferon gamma (INFY), leukotriene B4 receptor (LTB4R), MMP9, RON tyrosine kinase receptor (RON), miR-155, and miR-21[14, 18-20]. Total RNA from the induced sputum was extracted using TRIzol reagent (Sigma-Aldrich, MO, USA) following the manufacturer's protocol and reverse transcribed into cDNA using a PrimeScript RT reagent kit (Takara Bio, Shiga, Japan). qRT-PCR analysis was performed using SYBR Premix Ex Taq (Takara Bio) with an Applied Biosystems 7500 Real-Time PCR System using the following three-step cycling programs according to the instructions. The relative expression of target genes was normalized to that of GAPDH, and the expression levels of microRNAs were normalized to that of $\mathrm{U} 6 \mathrm{using}$ the $2^{-\triangle} \triangle \mathrm{Ct}$ method. The primers were as follows: GAPDH (forward primer 5'-GCGAGATCCCTCCAAAATCAA-3', reverse primer 5'-GTTCAC ACCCATGACGAACAT-3'); IFNy (forward primer 5'-GAGTGTGGA GACCATCAAGGAA-3', reverse primer 5'TGCGTTGGACATTCAA GTCAG-3'); MMP9 (forward primer 5'-TCATCTT CCAAGGCCAATCC-3', reverse primer 5'-GC AGAAGCCGAAGAGCTTGT-3'); CC16 (forward primer 5'-GGTCACACTGGCTCTCTGC T-3', 
reverse primer 5'-CATGGCAGCCTCATAACTG G-3'); LTB4R (forward primer 5'-ACCTGGCCGTATTGCTCAC T3', reverse primer 5'-GCTGG CGTACATGCTGACTC-3'); RON (forward primer 5'-

ATGAATGTGCGTCCAGAACA-3', reverse primer 5'-CAGGTCCAGCCCAAGAAC TA-3'); A1AR (forward primer 5'-GATCCTCTC CTTCGTGGTG-3', reverse primer 5'-CCCACACAAAGAAGTTGAAG-3'); miR-155 (forward primer 5'-CGCGTTAATGCTAATCGTGATAGG GGT); miR-21 (forward primer 5'-GCGCGTAG CTTATCAGACTGATGTTGA).

\subsection{Enzyme-linked immunosorbent assay (ELISA) assay}

Commercial ELISA kits were used to measure the inflammatory cytokines in the induced sputum supernatants. The measured inflammatory cytokines were CC16, TNF-a, IL-1 $\beta$, IL-6, IL-8, interleukin-10 (IL10), interleukin-17 (IL-17), granulocyte-macrophage colony stimulating factor (GM-CSF), interferon gamma (INFY), MCP-1, macrophage inflammatory protein 1a (MIP1a), vascular endothelial growth factor (VEGF), soluble CD40 ligand (sCD40 L) and SP-D [8, 21, 22]. The procedures were performed according to the manufacturer's instructions. Each 10- $\mu \mathrm{L}$ sample was prepared for analysis. The detection was performed at $450 \mathrm{~nm}$ using a multiscan spectrum.

\subsection{Statistical analysis}

Data are expressed as the means \pm SDs. Pearson's correlation was used for the correlation analyses. Data were analyzed using Pearson's test for normality and nonparametric Mann-Whitney $U$ tests or parametric unpaired t-tests. Correlations between variables were analyzed with Spearman's rank correlation analysis. All data analyses were performed using GraphPad Prism 6 (GraphPad Software, Inc., San Diego, CA). Statistical significance was indicated by a $p$ value $<.05$.

\section{Results}

\subsection{Characteristics of the study population}

Data on spirometry were available for 102 participants, and the characteristics of the study population are presented in Table 1. Sex but not age was significantly different between the mild and moderate group and the severe and extremely severe group. There were fewer women in the severe and extremely severe group. Neither symptoms nor complications differed between the two groups. An higher SGRQ score was found in the severe and extremely severe group than in the mild and moderate group. With regard to treatments, more treatment with inhaled corticosteroids (ICS) + long-acting $\beta$-agonist (LABA) + long-acting muscarinic antagonist (LAMA) was found in the severe and extremely severe group than in the mild and moderate group, while the opposite result was found for monobronchodilator therapy. No significant difference was found in treatments of dual bronchodilator therapy and ICS + LABA/ICS + LAMA therapy. There were no differences in acute exacerbations in the last year or the FeNO between the two groups. 
Table 1

Clinical characteristics of participants.Values are expressed as mean $\pm S D$, percentages, or sum.

\begin{tabular}{|c|c|c|c|}
\hline Characteristics & $\begin{array}{l}\text { mild and moderate } \\
\text { (FEV1\%pred } \geq 50 \% \text { ) }\end{array}$ & $\begin{array}{l}\text { severe and extremely } \\
\text { severe } \\
\text { (FEV1\%pred冈50\%) }\end{array}$ & $\begin{array}{l}\text { p- } \\
\text { value }\end{array}$ \\
\hline Number & $n=57$ & $\mathrm{n}=45$ & \\
\hline Men $₫ N / \%)$ / Women (N/\%) & $\begin{array}{l}42(73.7 \%) / 15 \\
(26.3 \%)\end{array}$ & $41(91.1 \%) / 4(8.9 \%)$ & 0.025 \\
\hline Age (years) & $65.4 \pm 7.3$ & $66.7 \pm 5.9$ & 0.357 \\
\hline \multicolumn{4}{|l|}{ Symptoms } \\
\hline - Cough & $30(52.6 \%)$ & $22(48.9 \%)$ & 0.707 \\
\hline - Expectoration & $37(64.9 \%)$ & $34(75.6 \%)$ & 0.246 \\
\hline - Polypnea & $38(66.7 \%)$ & $33(73.3 \%)$ & 0.467 \\
\hline \multicolumn{4}{|l|}{ Complications $(n=106)$} \\
\hline - Hypertension & $20(35.1 \%)$ & $16(35.6 \%)$ & 0.961 \\
\hline - Diabetes & $8(14.0 \%)$ & $4(8.9 \%)$ & 0.423 \\
\hline - Arrhythmia & $6(10.5 \%)$ & $4(8.9 \%)$ & 0.782 \\
\hline - Coronary Artery Disease & $5(8.8 \%)$ & $3(6.7 \%)$ & 0.204 \\
\hline - Hyperlipidemia & $3(5.3 \%)$ & $1(2.2 \%)$ & 0.695 \\
\hline - Cardiac Dysfunction & $2(3.5 \%)$ & $0(0 \%)$ & 0.422 \\
\hline - Other complications & $15(26.3 \%)$ & $9(20 \%)$ & 0.455 \\
\hline SGRQ & $25.9 \pm 17.1$ & $35.9 \pm 17.1$ & 0.004 \\
\hline $\mathrm{mMRC}$ & $0.94 \pm 0.95$ & $1.05 \pm 0.7$ & 0.296 \\
\hline CAT & $17.5 \pm 5.4$ & $19.7 \pm 6.5$ & 0.119 \\
\hline FEV1\%FVC & $64.0 \pm 8.0$ & $49.5 \pm 8.4$ & 0.000 \\
\hline FEV1 \% pred & $67.4 \pm 12.0$ & $39.0 \pm 8.9$ & 0.000 \\
\hline FeNO (ppb) & $33.8 \pm 32.3$ & $28.4 \pm 22.3$ & 0.270 \\
\hline \multicolumn{4}{|l|}{ Treatments } \\
\hline - Mono Bronchodilator & $19(33.3 \%)$ & $6(13.3 \%)$ & 0.020 \\
\hline - Dual Bronchodilator & $4(7.0 \%)$ & $8(17.8 \%)$ & 0.094 \\
\hline - ICS+LABA/ICS+LAMA & $14(24.6 \%)$ & $8(17.8 \%)$ & 0.408 \\
\hline
\end{tabular}




\begin{tabular}{|c|c|c|}
\hline - ICS+LABA+LAMA & $16(28.1 \%)$ & $23(51.1 \%)$ \\
\hline - No Inhaled Medication & $4(7.0 \%)$ & $0(0 \%)$ \\
\hline $\begin{array}{l}\text { Acute Exacerbation (over the past } \\
\text { year) }\end{array}$ & $10(17.5 \%)$ & $14(31.1 \%)$ \\
\hline \multicolumn{3}{|c|}{$\begin{array}{l}\text { Abbreviation: SGRQ, St George's Respiratory Questionnaire; mMRC, modified Medical Research } \\
\text { Council; CAT, COPD Assessment Test; FEV1\%FVC, forced expiratory volume in } 1 \text { second \% force } \\
\text { capacity; FEV1\%pred, percentage of forced expiratory volume in one second in the predicted val } \\
\text { FeNO, fraction exhaled nitric oxide; ICS, inhaled corticosteroid; LABA, long-acting } \beta \text {-agonist; LAM } \\
\text { long-acting muscarinic antagonist. }\end{array}$} \\
\hline
\end{tabular}

A total of 54 sputum samples were deemed acceptable for inclusion in the cell component analysis. The cellular compositions of the induced sputum samples from COPD patients are shown in Fig. 2 . The percentage of eosinophils $(p=0.048)$ was significantly higher in the severe and extremely severe group than in the mild and moderate group, although there were no significant differences between the two groups in the percentages of neutrophils $(p=0.568)$, lymphocytes $(p=0.545)$ or monocytes $(p=0.073)$. Four inflammatory phenotypes were assessed based on the proportions of sputum neutrophils and eosinophils. The results showed similar percentages of the neutrophil phenotype $(p=0.793)$, eosinophil phenotype $(p=0.702)$, mixed granulocyte phenotype $(p=0.069)$ and paucigranulocytic phenotype $(p=$ 0.068 ) in the mild and moderate group and the severe and extremely severe group.

\subsection{Inflammatory biomarker levels in the induced sputum from COPD patients}

As shown in Fig. 3, compared with the mild and moderate group, the mRNA levels of $\operatorname{CC16}(p=0.013)$ and miR-155 ( $p=0.017)$ were significantly lower and the mRNA level of LTB4R $(p=0.033)$ and MMP9 $(p=$ $0.012)$ were significantly higher in the severe and extremely severe group; there was no difference in A1AR $(p=0.064)$, INFY $(p=0.389)$, RON $(p=0.148)$ or miR-21 $(p=0.905)$ between the two groups. The levels of 13 inflammatory mediators, namely, TNF-a $(p=0.214)$, IL-1 $\beta(p=0.049)$, IL-6 $(p=0.280)$, IL-8 $(p=0.432)$, IL-10 $(p=1.000), \mathrm{IL}-17(p=0.772), \operatorname{GM}-\mathrm{CSF}(p=0.564), \mathrm{IFNY}(p=0.811), \operatorname{MCP}-1 \quad(p=0.919), \operatorname{MIP} 1 \mathrm{a}(p=$ $0.580), \operatorname{VEGF}(p=0.700), \operatorname{sCD} 40 \mathrm{~L}(p=1.000), \operatorname{CC16}(p=0.263)$, and SP-D $(p=0.897)$, were detected and are presented in Fig. 4. Overall, there were few differences in any sputum inflammatory cytokines, and only IL-1 $\beta$ showed a significant difference, with a relatively lower level in the severe and extremely severe group.

\subsection{Correlation of inflammatory biomarker levels in induced sputum and lung function by simple linear regression}

The associations between gene mRNA expression, airway inflammatory cytokines and cell phenotypes in the induced sputum samples and FEV1\%pred or FEV1\%FVC were analyzed with simple linear regression. As shown in Table 2, SGRQ $(p=0.025, p=0.044)$ and the percentage of eosinophils $(p=0.031, p=0.039)$ 
had negative correlations and CC16 ( $p=0.004, p=0.008)$ had a positive correlation with the two lung function parameters (FEV1\%pred and FEV1\%FVC). CAT $(p=0.030)$ was negatively associated with FEV1\%pred, and IL-1 $(p=0.046)$ was positively associated with FEV1\%FVC. Otherwise, no significant associations were found between other induced sputum inflammatory mediators or cell subtypes and the lung function parameters.

Table 2

Correlation between gene mRNA expression, airway inflammation markers or cell subtypes in induced sputum and different evaluation system or lung function by simple linear regression analysis.

\begin{tabular}{|c|c|c|c|c|}
\hline & FEV1\%pred & p-value & FEV1\%FVC & p-value \\
\hline SGRQ & -0.217 & 0.025 & -0.121 & 0.044 \\
\hline CAT & -0.581 & 0.086 & -0.446 & 0.030 \\
\hline \multicolumn{5}{|c|}{$\begin{array}{l}\text { mRNA related expression in } \\
\text { induced sputum cells }\left(2^{-\triangle \triangle \mathrm{CT}}\right)\end{array}$} \\
\hline MMP9 & -7.743 & 0.190 & -3.462 & 0.321 \\
\hline CC16 & 11.558 & 0.004 & 6.434 & 0.008 \\
\hline LTB4R & -4.746 & 0.359 & -1.802 & 0.554 \\
\hline miR-155 & 5.509 & 0.063 & 3.304 & 0.059 \\
\hline \multicolumn{5}{|c|}{$\begin{array}{l}\text { Airway inflammation cytokines } \\
\text { in sputum supernatant }(\mathrm{pg} / \mathrm{mL})\end{array}$} \\
\hline IL-1 $\beta$ & 11.669 & 0.046 & 6.213 & 0.091 \\
\hline \multicolumn{5}{|c|}{ Cell phenotypes in induced sputum (\%) } \\
\hline Neutrophils & -9.041 & 0.456 & -4.993 & 0.492 \\
\hline Eosinophils & -108.468 & 0.031 & -62.283 & 0.039 \\
\hline Lymphocytes & -8.585 & 0.744 & 3.291 & 0.834 \\
\hline Monocytes & 28.911 & 0.072 & 12.353 & 0.202 \\
\hline \multicolumn{5}{|c|}{$\begin{array}{l}\text { Abbreviation: FEV1\%pred, percentage of forced expiratory volume in one second in the predicted } \\
\text { value; FEV1\%FVC, forced expiratory volume in } 1 \text { second \% forced vital capacity; SGRQ, St George's } \\
\text { Respiratory Questionnaire; CAT, COPD Assessment Test; MMP-9, matrix metalloproteinase 9; CC16, } \\
\text { Clara cell 16; LTB4R, leukotriene B4 receptor; miR-155, microRNA-155; IL-1 } 13 \text {, interleukin-1 } \beta\end{array}$} \\
\hline
\end{tabular}

\subsection{Correlation of inflammatory biomarker levels in induced sputum and lung function by multiple linear regression}

Furthermore, the independent associations of mRNA expression in the induced sputum cells and the levels of inflammatory cytokines in the supernatant of the induced sputum samples with the lung 
function parameters were investigated with multiple linear regression. As shown in Fig. 5, only CC16 mRNA expression in the induced sputum cells was significantly positively correlated with both FEV1\%pred $(p=0.005)$ and FEV1\%FVC $(p=0.008)$. The expression of miR-155 $(p=0.046)$ in the induced sputum cells was positively associated with FEV1\%FVC but not FEV1\%pred after removing confounding factors that were significantly associated with pulmonary function in the univariate model, including age and sex. No significant association between other inflammatory mediators and FEV1\%pred or FEV1\%FVC was found in our study.

\subsection{Predictive value of sputum cell CC16 mRNA expression for the severity of lung function decline in COPD}

According to the ROC curve, a relative expression level of CC16 mRNA in the induced sputum cells of 1.09 was observed to be the diagnostic threshold for a decrease in COPD lung function (FEV1\%pred). The sensitivity and specificity for the severity of COPD were $55.6 \%$ and $88.2 \%$, respectively, and the area under the curve (AUC) was 0.725. (Fig. 6)

\section{Discussion}

In the present study, a large number of inflammatory cells and inflammatory biomarkers in induced sputum were analyzed, and their association with pulmonary function in patients with COPD were determined. Some of the observations build on previous findings and might provide new sputum inflammatory biomarkers that can be used to predict the severity of lung function decline in patients with COPD.

Studies have reported that the larger the proportion of serum eosinophils is, the higher the risk of COPD exacerbation, suggesting that the level of eosinophils in the peripheral blood could be used as a biological indicator of the prognosis of the disease[9]. However, it was found that peripheral eosinophil counts did not accurately predict sputum eosinophil counts, especially in patients with mild-to-moderate COPD [23]. In steroid-naïve patients with mild-to-moderate COPD, no relationship between the peripheral eosinophil count and the sputum eosinophil percentage was found, highlighting the importance of assessing the level of eosinophils in the airways [6]. Our study focused on the relationship between local eosinophilic airway inflammation and lung function. As expected, the sputum eosinophil percentage was negatively correlated with FEV1\%pred. Annette T Hastie et al. reported that a high concentration of sputum eosinophils was a better biomarker than a high concentration of blood eosinophils for the COPD patient subgroup with more severe disease, more frequent exacerbations, and a higher proportion of patients with emphysema[23]. According to the current criteria, a high level of blood eosinophilia has been defined by a blood eosinophil threshold $\geq 2 \%$ or $\geq 300$ cells/ $\mu L[24,25]$. COPD patients with high levels of blood eosinophils have been found to be more sensitive to treatment with ICS, and there was an increase in the number of exacerbations in these patients when ICS therapy was discontinued[26]. Across studies, the incidence of high blood eosinophil levels varies significantly, ranging from $14-37 \%$ of COPD 
patients, while high levels of sputum eosinophils were detected in $30 \%$ 40\% of COPD patients, suggesting that sputum eosinophils may an effective and more stable predictor of the response to ICS therapy in COPD patients[6,27]. It is important for clinical trials targeting eosinophilic inflammation in COPD to consider the assessment of sputum eosinophils. However, our study showed that there were no correlations of the percentages of neutrophils, lymphocytes or monocytes in induced sputum samples with lung function decrease in patients with COPD.

CC16 is a protein secreted by Clara cells in the bronchioles and terminal bronchioles; it is specific to the lung tissue and exerts anti-inflammatory effects[28]. Recent studies have shown that epithelial cell damage and airway remodeling result in the reduction of the level of CC16 in patients with COPD[29]. Most previous studies have indicated that serum CC16 levels are related to functional declines in COPD patients; however, few studies have focused on sputum CC16 levels[30, 31]. We found a significantly lower level of CC16 mRNA in patients with severe and extremely severe COPD than in those with mild and moderate COPD, suggesting that that CC16 in the lower airways may contribute to the progressive anatomical damage in the end stage of COPD. Moreover, multiple regression analysis showed that the level of CC16 mRNA in the induced sputum cells was independently correlated with the decline in lung function (FEV1\%pred and FEV1\%FVC). The report from David Chi-Leung Lam et al. showed a relationship between low serum CC16 levels at baseline and greater lung function decline over the course of 10 years in a Chinese longitudinal cohort. In that study, they also found that the level of CC16 mRNA in endoscopic biopsies of the bronchial epithelium was also correlated with the FEV1/FVC ratio[30]. Compared to endoscopic biopsies of bronchial epithelium, the induction of sputum production and subsequent detection of CC16 mRNA in our study was less invasive and more practical. Furthermore, based on our data, when the relative mRNA level of CC16 in the induced sputum cells was less than 1.09, it indicated that the patient may have severely limited airflow, which suggested that the CC16 mRNA level in the induced sputum cells may be a predictive biomarker for the severity of COPD.

MiRNAs are 19-22 nucleotides in length and regulate target mRNA translation or degradation. miRNAs play crucial roles in apoptosis, cell differentiation, proliferation and other biological processes [32]. A few studies have shown that the dysregulation of several miRNAs is involved in the inflammatory response and fibrosis in patients with pulmonary disease[14]. Based on the findings in earlier studies, we chose to detect the expression levels of MiR-155 and MiR-21 in the induced sputum cells from patients with COPD. Our study showed lower levels of miR-155 but not miR-21 in the severe and extremely severe group than in the mild and moderate group. Furthermore, miR-155 was independently positively correlated with the FEV1/FVC ratio but not FEV1\%pred. Different results regarding miR-155 levels in patients with COPD have been reported in several previous studies, while in patients with eosinophilic-related lung disease, the miR155 levels were found to be reduced[14, 32, 33]. In fact, miR-155 was reported to regulate Th cell differentiation, which causes a decrease in the level of eosinophils and the release of inflammatory cytokines, including IL-6 [34]. The increased percentage of eosinophils but not neutrophils associated with more severe COPD in our analysis might be related to miR-155 downregulation. 
In addition to the crucial findings reported above, the mRNA levels of MMP9 and LTB4R were significantly higher in the induced sputum samples from the severe and extremely severe group than in those from the mild and moderate group. Studies have shown that MMP-9, a glycosylated gelatinase, increases the deposition of extracellular matrix by mediating the accumulation and infiltration of inflammatory cells in the lung, resulting in pulmonary vascular bed remodeling and lung tissue damage in patients with COPD[11]. Mitsunobu et al. reported that LTB4 levels were elevated in the peripheral blood or induced sputum of COPD patients, and these elevated levels could activate the inflammatory response by combining with LTB4R. The increase in LTB4R in the induced sputum samples of patients with severe and extremely severe COPD in our study provided evidence of LTB4-related inflammation[35]. Among the inflammatory cytokines that we measured, only the reduced level of IL- $\beta$ achieved statistical significance. In fact, the levels of many of the inflammatory mediators in the induced sputum supernatant were below the level of detection for commercial ELISA kits. The use of dithiothreitol to process the induced sputum samples has been reported to decrease the concentrations of many proteins, including inflammatory cytokines[36-38]. Other factors, including lysis time and temperature, affect the concentrations of the inflammatory biomarker in the induced sputum supernatant. Studies have shown that the measurement of mRNA expression may be a more sensitive method of evaluating inflammatory mediators in induced sputum[7, 39, 40]. In accordance with this, our analysis also showed that sputum cell CC16 mRNA but not protein expression in the sputum supernatant was closely and independently correlated with lung function decline (FEV1\%pred and FEV1\%FVC) in patients with COPD. Thus, we believe that the assessment of induced sputum cells by qRT-PCR could be a feasible and stable method for the clinical determination of the severity of COPD.

The limitations of this study include the lack of data on parameters in the serum of COPD patients; therefore, we were unable to investigate the relationships between and differences in inflammatory biomarker levels in the sputum and the blood. Future studies should be performed to determine the correlations of inflammatory mediators involved in local airway inflammation and systemic inflammation. Second, only the levels of mRNA for inflammatory biomarkers in sputum cells were measured. Studies to confirm the protein levels in sputum cells and their associations with the pulmonary function parameters are still needed. Furthermore, since the sample size was small, the results may be biased, and this sample population is not representative of the entire COPD population.

\section{Conclusion}

In conclusion, we demonstrated that the levels of eosinophils, MMP9 mRNA, LTB4R mRNA, CC16 mRNA and miR-155 in induced sputum samples are associated with the severity of COPD. The induced sputum cell CC16 mRNA expression level was the only biomarker that was positively and independently associated with both FEV1\%pred and FEV\%FVC, indicating that it has the potential as a novel biomarker that can be used to monitor the severity of the decline in lung function in COPD patients. Compared to the analysis of the sputum supernatant, the analysis of the sputum cells appeared to be more reliable, which suggests that sputum cells should be considered in the analysis of the severity of COPD in clinical practice. 


\section{Abbreviations}

COPD: chronic obstructive pulmonary disease; SGRQ: St George's Respiratory Questionnaire; mMRC: modified Medical Research Council; CAT: COPD Assessment Test; FEV1\%FVC: forced expiratory volume in 1 second \% forced vital capacity; FEV1\%pred: percentage of forced expiratory volume in one second in the predicted value; FeNO: fraction exhaled nitric oxide; ICS: inhaled corticosteroid; LABA: long-acting $\beta$ agonist; LAMA: long-acting muscarinic antagonist; TNF-a: tumor necrosis factor $\alpha$; IL-1 $\beta$ : interleukin-1 $\beta$; IL-6: interleukin-6; IL-8: interleukin-8; LTB4: leukotriene B4; MCP-1: monocyte chemotactic protein-1; MMP9: matrix metalloproteinase 9; CC16: Clara cell 16; SP-D: surfactant protein-D; A1AR: A1 adenosine receptor; INFY: interferon gamma; LTB4R: leukotriene B4 receptor; miR-155: microRNA-155; miR-21: microRNA-21.

\section{Declarations}

\section{Author contributions}

Conceived of and designed the study: Chen ZH, Li HY. Performed the biological experiments: He YT, Deng Z, Jin JJ, Mao RL, Gao L, Zhang Y. Performed the statistical analyses: He YT, Deng Z, Wang G, Gao P, Xie M. Wrote and modified the paper: He YT, Chen ZH, Wang G, Gao P, Xie M. All authors read and approved the final manuscript.

\section{Consent for publication}

Not applicable.

\section{Funding}

This work was supported by the National Natural Science Foundation of China (81470211 and 81970023 to ZHC), the Shanghai Health Committee (201840288), the Shanghai Respiratory Research Institute and Young Scientists Training Program of Zhongshan Hospital, the Shanghai Top-Priority Clinical Key Disciplines Construction Project (2017ZZ02013) and the Shanghai Municipal Key Clinical Specialty (shslczdzk02201).

\section{Conflicts of interest}

The authors declare that they have no conflicts of interest.

\section{Ethics approval and consent to participate}


All samples were obtained with the written consent of patients. The study was approved by the ethics committee of Zhongshan Hospital Fudan University.

\section{Availability of data and materials}

The datasets used and/or analysed during the current study are available from the corresponding author on reasonable request.

\section{References}

1. Vestbo, J., et al., Global strategy for the diagnosis, management, and prevention of chronic obstructive pulmonary disease: GOLD executive summary. Am J Respir Crit Care Med, 2013. 187(4): p. 347-65.

2. Tantucci, C. and D. Modina, Lung function decline in COPD. Int J Chron Obstruct Pulmon Dis, 2012. 7: p. 95-9.

3. Lopez-Campos, J.L., W. Tan, and J.B. Soriano, Global burden of COPD. Respirology, 2016. 21(1): p. 14-23.

4. Odeyemi, Y.E., et al., Does Low FEV1 in Addition to Fixed Ratio and/or Lower Limit of Normal of FEV1/FVC Improve Prediction of Mortality in COPD? The NHANES-III-linked-mortality Cohort. J Natl Med Assoc, 2019. 111(1): p. 94-100.

5. Wang, Y., et al., Role of inflammatory cells in airway remodeling in COPD. Int J Chron Obstruct Pulmon Dis, 2018. 13: p. 3341-3348.

6. Proboszcz, M., et al., Relationship between Blood and Induced Sputum Eosinophils, Bronchial Hyperresponsiveness and Reversibility of Airway Obstruction in Mild-to-Moderate Chronic Obstructive Pulmonary Disease. COPD, 2019. 16(5-6): p. 354-361.

7. Paplińska-Goryca, M., et al., Expression of Inflammatory Mediators in Induced Sputum: Comparative Study in Asthma and COPD, in Clinical Research Involving Pulmonary Disorders. 2016. p. 101-112.

8. Lin, X., et al., Correlation Between Tumor Necrosis Factor-alpha and Interleukin-1beta in Exhaled Breath Condensate and Pulmonary Function. Am J Med Sci, 2017. 354(4): p. 388-394.

9. Tashkin, D.P. and M.E. Wechsler, Role of eosinophils in airway inflammation of chronic obstructive pulmonary disease. International Journal of Chronic Obstructive Pulmonary Disease, 2018. Volume 13: p. 335-349.

10. Zeng, Y.-Y., et al., Altered serum levels of type I collagen turnover indicators accompanied by IL-6 and IL-8 release in stable COPD. International Journal of Chronic Obstructive Pulmonary Disease, 2019. Volume 14: p. 163-168.

11. Hao, W., et al., Inflammatory mediators in exhaled breath condensate and peripheral blood of healthy donors and stable COPD patients. Immunopharmacology and Immunotoxicology, 2019. 41(2): p. 224-230. 
12. Lomas, D.A., et al., Evaluation of serum CC-16 as a biomarker for COPD in the ECLIPSE cohort. Thorax, 2008. 63(12): p. 1058-1063.

13. Lomas, D.A., et al., Serum surfactant protein D is steroid sensitive and associated with exacerbations of COPD. Eur Respir J, 2009. 34(1): p. 95-102.

14. Kara, M., G. Kirkil, and S. Kalemci, Differential Expression of MicroRNAs in Chronic Obstructive Pulmonary Disease. Adv Clin Exp Med, 2016. 25(1): p. 21-6.

15. Rosenberg, S.R. and R. Kalhan, Biomarkers in chronic obstructive pulmonary disease. Transl Res, 2012. 159(4): p. 228-37.

16. Tsikrika, S., et al., The role of non-invasive modalities for assessing inflammation in patients with non-cystic fibrosis bronchiectasis. Cytokine, 2017. 99: p. 281-286.

17. Aurora, P., Multiple-breath inert gas washout test and early cystic fibrosis lung disease. Thorax, 2010. 65(5): p. 373-374.

18. Sun, C.-X., et al., A protective role for the A1 adenosine receptor in adenosine-dependent pulmonary injury. Journal of Clinical Investigation, 2005. 115(1): p. 35-43.

19. Dahl, R., et al., Effects of an oral MMP-9 and -12 inhibitor, AZD1236, on biomarkers in moderate/severe COPD: A randomised controlled trial. Pulmonary Pharmacology \& Therapeutics, 2012. 25(2): p. 169-177.

20. Braido, F., et al., Clara cell 16 protein in COPD sputum: a marker of small airways damage? Respir Med, 2007. 101(10): p. 2119-24.

21. Zemans, R.L., et al., Multiple biomarkers predict disease severity, progression and mortality in COPD. Respiratory Research, 2017. 18(1).

22. Eickmeier, O., et al., Sputum biomarker profiles in cystic fibrosis (CF) and chronic obstructive pulmonary disease (COPD) and association between pulmonary function. Cytokine, 2010. 50(2): p. 152-157.

23. Hastie, A.T., et al., Association of sputum and blood eosinophil concentrations with clinical measures of COPD severity: an analysis of the SPIROMICS cohort. The Lancet Respiratory Medicine, 2017. 5(12): p. 956-967.

24. SIMPSON, J.L., R. SCOTT, and M.J. BOYLE, inflammatory subtypes in asthma:Assessment and identification using induced sputum Respirology, 2006. 11: p. 54-61.

25. Watz, H., et al., Blood eosinophil count and exacerbations in severe chronic obstructive pulmonary disease after withdrawal of inhaled corticosteroids: a post-hoc analysis of the WISDOM trial. The Lancet Respiratory Medicine, 2016. 4(5): p. 390-398.

26. Cheng, S.-L. and C.-H. Lin, Effectiveness using higher inhaled corticosteroid dosage in patients with COPD by different blood eosinophilic counts. International Journal of Chronic Obstructive Pulmonary Disease, 2016. Volume 11: p. 2341-2348.

27. Saha, S. and C.E. Brightling, Eosinophilic airway inflammation in COPD. Int J Chron Obstruct Pulmon Dis, 2006. 1(1): p. 39-47. 
28. Laucho-Contreras, M.E., et al., Protective role for club cell secretory protein-16 (CC16) in the development of COPD. European Respiratory Journal, 2015. 45(6): p. 1544-1556.

29. Nanson, C.J., et al., Exercise alters serum pneumoprotein concentrations. Respir Physiol, 2001. 127(23): p. 259-65.

30. Lam, D.C., et al., CC16 levels correlate with cigarette smoke exposure in bronchial epithelial cells and with lung function decline in smokers. BMC Pulm Med, 2018. 18(1): p. 47.

31. Guerra, S., et al., Relation between circulating CC16 concentrations, lung function, and development of chronic obstructive pulmonary disease across the lifespan: a prospective study. The Lancet Respiratory Medicine, 2015. 3(8): p. 613-620.

32. Mahesh, G. and R. Biswas, MicroRNA-155: A Master Regulator of Inflammation. J Interferon Cytokine Res, 2019. 39(6): p. 321-330.

33. De Smet, E.G., et al., The role of miR-155 in cigarette smoke-induced pulmonary inflammation and COPD. Mucosal Immunology, 2019. 13(3): p. 423-436.

34. Malmhall, C., et al., MicroRNA-155 is essential for T(H)2-mediated allergen-induced eosinophilic inflammation in the lung. J Allergy Clin Immunol, 2014. 133(5): p. 1429-38, 1438 e1-7.

35. Dong, R., et al., Cigarette smoke-induced lung inflammation in COPD mediated via LTB4/BLT1/SOCS1 pathway. Int J Chron Obstruct Pulmon Dis, 2016. 11: p. 31-41.

36. Woolhouse, I.S., D.L. Bayley, and R.A. Stockley, Effect of sputum processing with dithiothreitol on the detection of inflammatory mediators in chronic bronchitis and bronchiectasis. Thorax, 2002. 57: p. 667-671.

37. Efthimiadis, A., et al., Induced sputum cell and fluid-phase indices of inflammation: comparison of treatment with dithiothreitol vs phosphate-buffered saline. European Respiratory Journal, 1997. 10(6): p. 1336-1340.

38. Elzbieta Grebski, M., D. Christer Peterson, and M. Tullio C. Medici, Effect of Physical and Chemical Methods of Homogenization on Inflammatory Mediators in Sputum of Asthma Patients. CHEST, 2001. 119: p. 1521-1525.

39. Gelder, C.M., P S Thomas, and D.H. Yates, Cytokine expression in normal, atopic, and asthmatic subjects using the combination of sputum induction and the polymerase chain reaction. Thorax, 1995. 50: p. 1033-1037.

40. Jung, J.-W., et al., Expression Levels of Eosinophil Granule Protein mRNAs in Induced Sputum Reflect Airway Hyperresponsiveness and Airflow Limitation. The Tohoku Journal of Experimental Medicine, 2014. 233(1): p. 49-56.

\section{Figures}




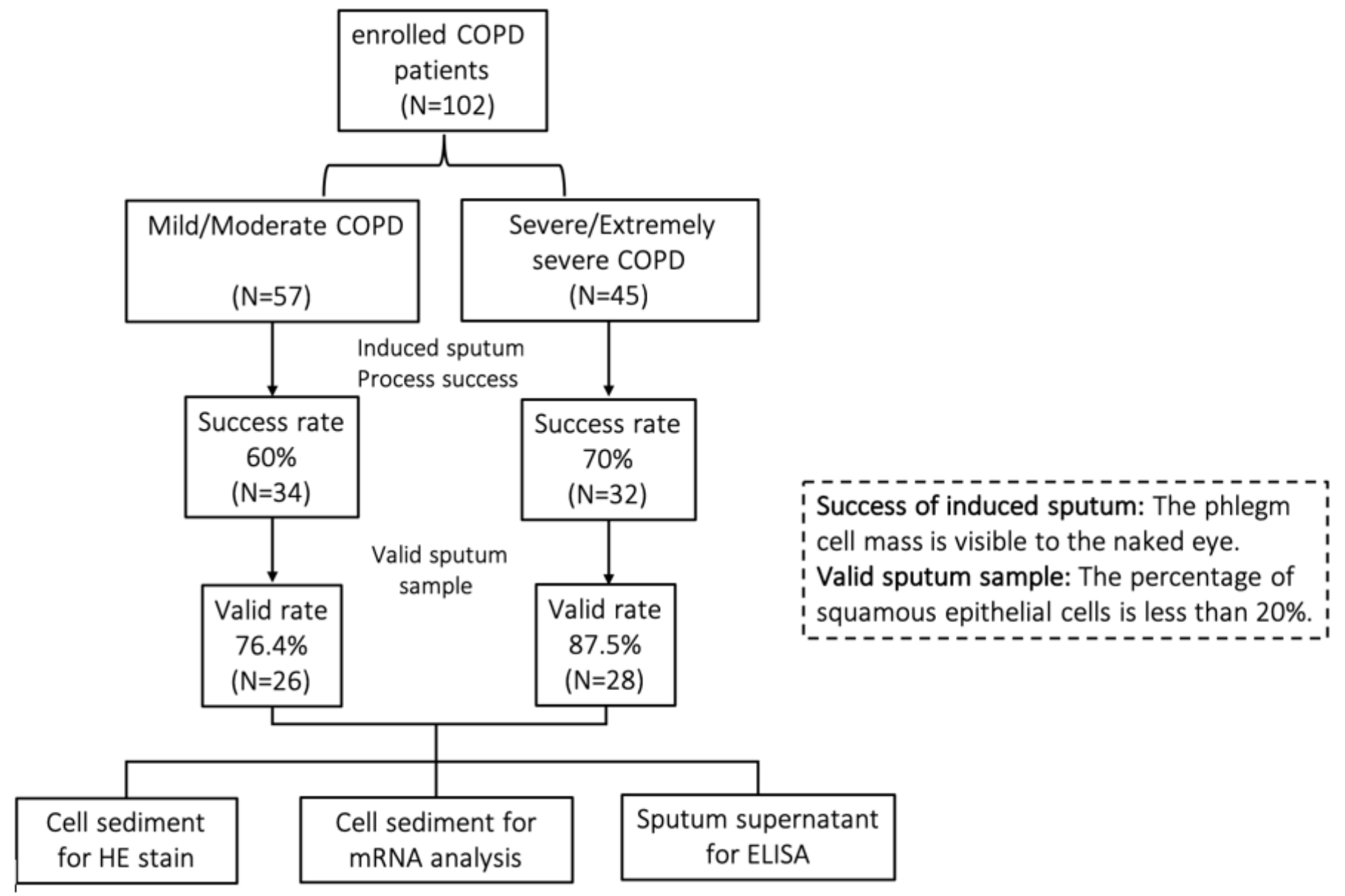

\section{Figure 1}

A flow chart of subject enrollment, induced sputum collection and assessment and specimen measurement.

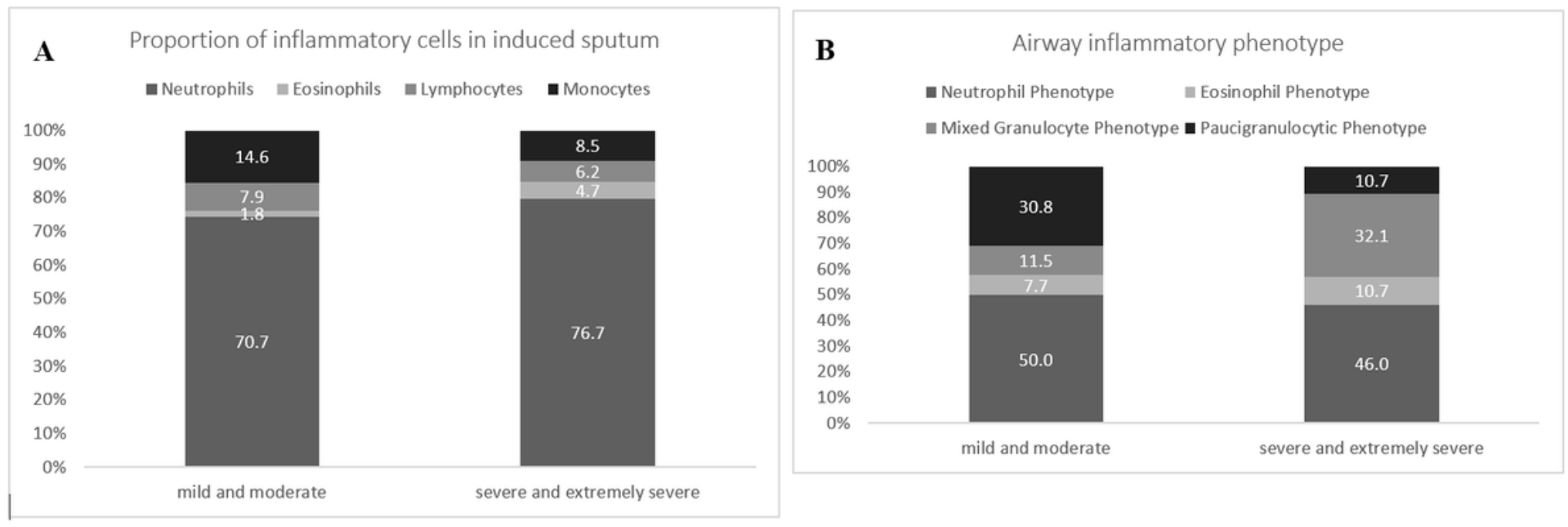

\section{Figure 2}

Sputum characteristics of the participants. A. The proportions of neutrophils $(p=0.568)$, eosinophils $(p=0.048)$, lymphocytes $(p=0.545)$ and monocytes $(p=0.073)$ in the induced sputum were evaluated and 
compared between the mild and moderate group and the severe and extremely severe group. $B$. The percentages classified as the neutrophil phenotype $(p=0.793)$, eosinophil phenotype $(p=0.702)$, mixed granulocyte phenotype $(p=0.069)$ and paucigranulocytic phenotype $(p=0.068)$ in the induced sputum were compared between the two groups. Neutrophilic phenotype, neutrophils $\geq 61 \%$ and eosinophils $<3 \%$; eosinophilic phenotype, neutrophils $<61 \%$ and eosinophils $\geq 3 \%$; mixed phenotype, neutrophils $\geq 61 \%$ and eosinophils $\geq 3 \%$; paucigranulocytic phenotype, neutrophils $<60 \%$ and eosinophils $<3 \%$. ${ }^{*} p<0.05$, significant compared to the mild and moderate group.

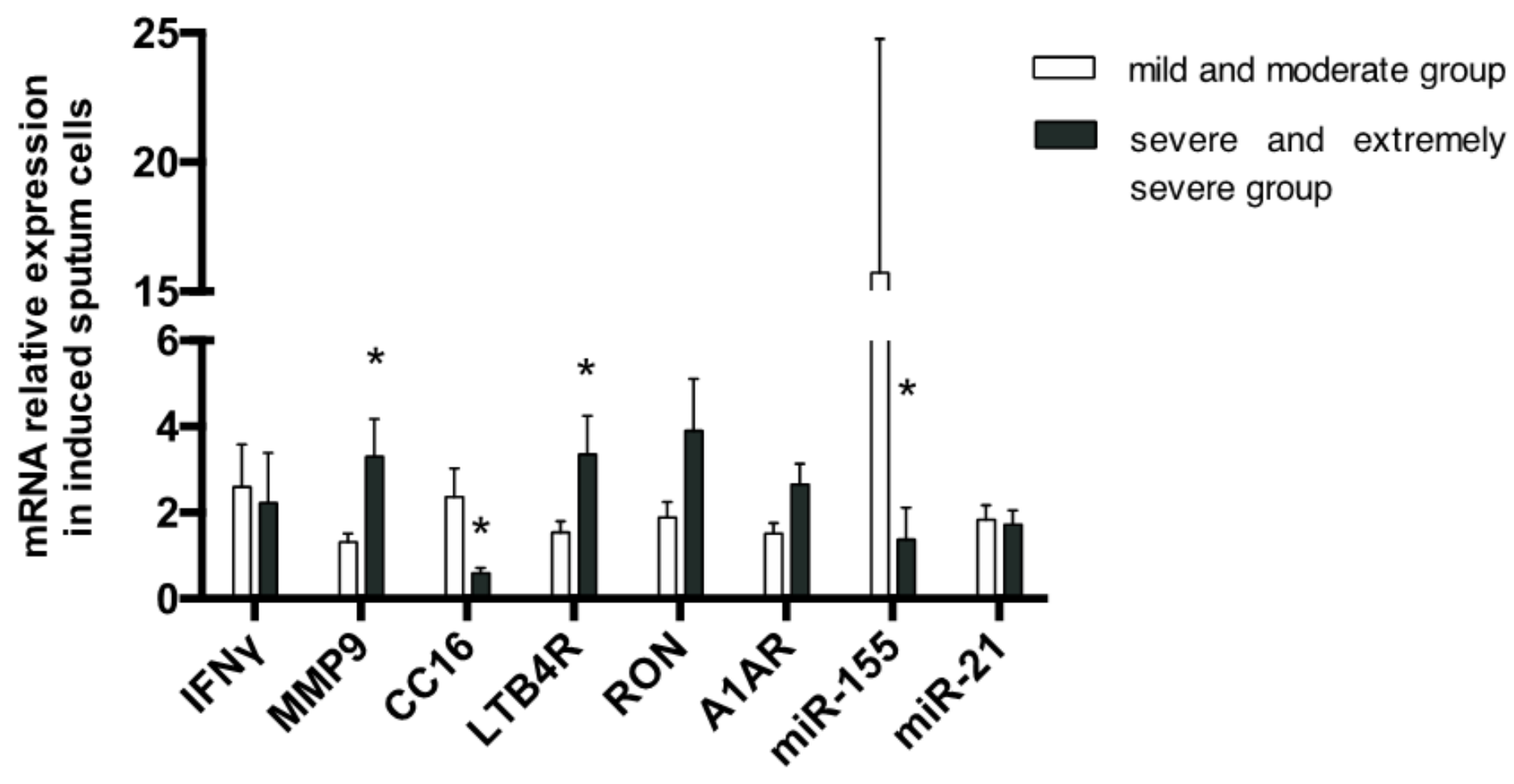

Figure 3

$\operatorname{A1AR}(p=0.064), \operatorname{CC16}(p=0.013), \operatorname{INFY}(p=0.389), \operatorname{LTB} 4 R(p=0.033), \operatorname{MMP9}(p=0.012), \operatorname{RON}(p=0.148)$, miR-155 ( $p=0.017)$, and miR-21 ( $p=0.905)$ mRNA expression in induced sputum cells compared between the mild and moderate group and the severe and extremely severe group. Data are expressed as the means \pm SEMs. ${ }^{*} p<0.05 \llbracket$ significant compared to the mild and moderate group. 


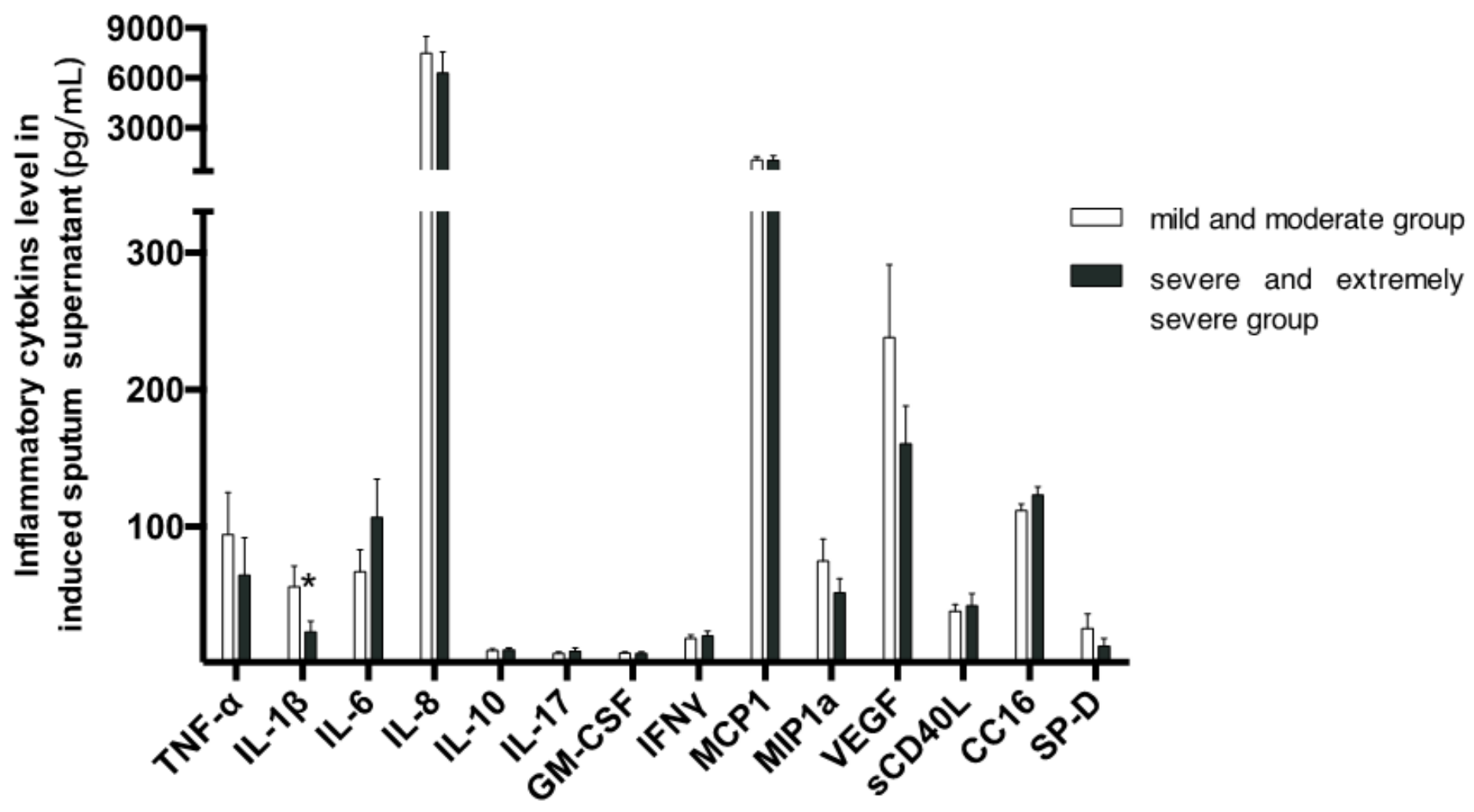

Figure 4

TNF- $a(p=0.214), I L-1 \beta(p=0.049), I L-6 \quad(p=0.280), I L-8 \quad(p=0.432), I L-10 \quad(p=1.000), I L-17 \quad(p=0.772), G M-C S F$ $(p=0.564), \operatorname{IFNY}(p=0.811), \operatorname{MCP}-1 \quad(p=0.919), \operatorname{MIP} 1 a(p=0.580), \operatorname{VEGF}(p=0.700), \operatorname{sCD} 40 \mathrm{~L}(p=1.000), \operatorname{CC} 16$ $(p=0.263)$, and SP-D $(p=0.897)$ levels in the induced sputum supernatant compared between the mild and moderate group and the severe and extremely severe group. Data are expressed as the means \pm SEMs. ${ }^{*} \mathrm{p}<0.05$, significant compared to the mild and moderate group. 

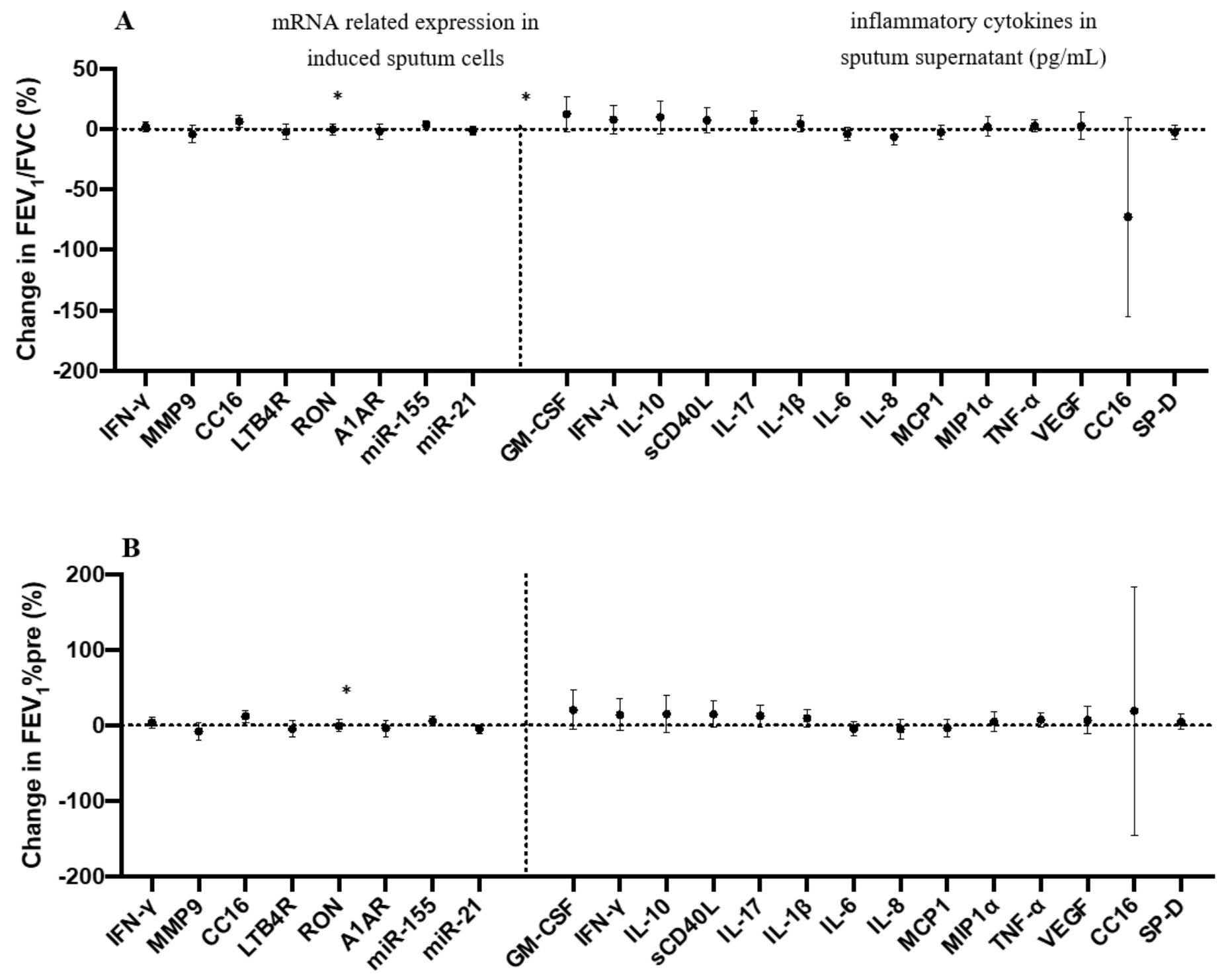

Figure 5

Associations of mRNA expression and inflammatory markers in the induced sputum with lung function by multiple linear regression. A. Correlations between FEV1/FVC (\%) and A1AR $(p=0.580), C C 16$ $(p=0.008), \operatorname{INFY}(p=0.306), \operatorname{LTB} 4 R(p=0.488), \operatorname{MMP9}(p=0.278), \operatorname{RON}(p=0.997), \operatorname{miR}-155(p=0.046), \operatorname{miR}-21$ $(p=0.545)$ mRNA expression and TNF- $\alpha(p=0.245), I L-1 \beta(p=0.181), I L-6(p=0.151), I L-8(p=0.077), I L-10$ $(p=0.145), I L-17(p=0.088), \operatorname{GM}-C S F(p=0.095), \operatorname{IFNY}(p=0.176), \operatorname{MCP}-1(p=0.419)$, MIP1a $(p=0.605)$, VEGF $(p=0.631), s C D 40 L(p=0.141), C C 16(p=0.080), S P-D(p=0.351)$ levels in the supernatant. B. Correlations between FEV1\%pred and A1AR ( $p=0.486)$, CC16 ( $p=0.005)$, INFY ( $p=0.293)$, LTB4R $(p=0.390)$, MMP9 $(p=0.209), R O N(p=0.881)$, miR-155 ( $p=0.078), \operatorname{miR}-21 \quad(p=0.129)$ mRNA expression and TNF- $a(p=0.102)$, IL-1 $(p=0.114), I L-6(p=0.370), I L-8(p=0.420), I L-10(p=0.214), I L-17(p=0.083), G M-C S F(p=0.118), I F N y$ $(p=0.169), \operatorname{MCP}-1 \quad(p=0.538), \operatorname{MIP1a}(p=0.438), \operatorname{VEGF}(p=0.418), \operatorname{sCD} 40 \mathrm{~L}(p=0.093), \operatorname{CC} 16(p=0.808)$, and SP-D $(p=0.325)$ levels in the sputum. 


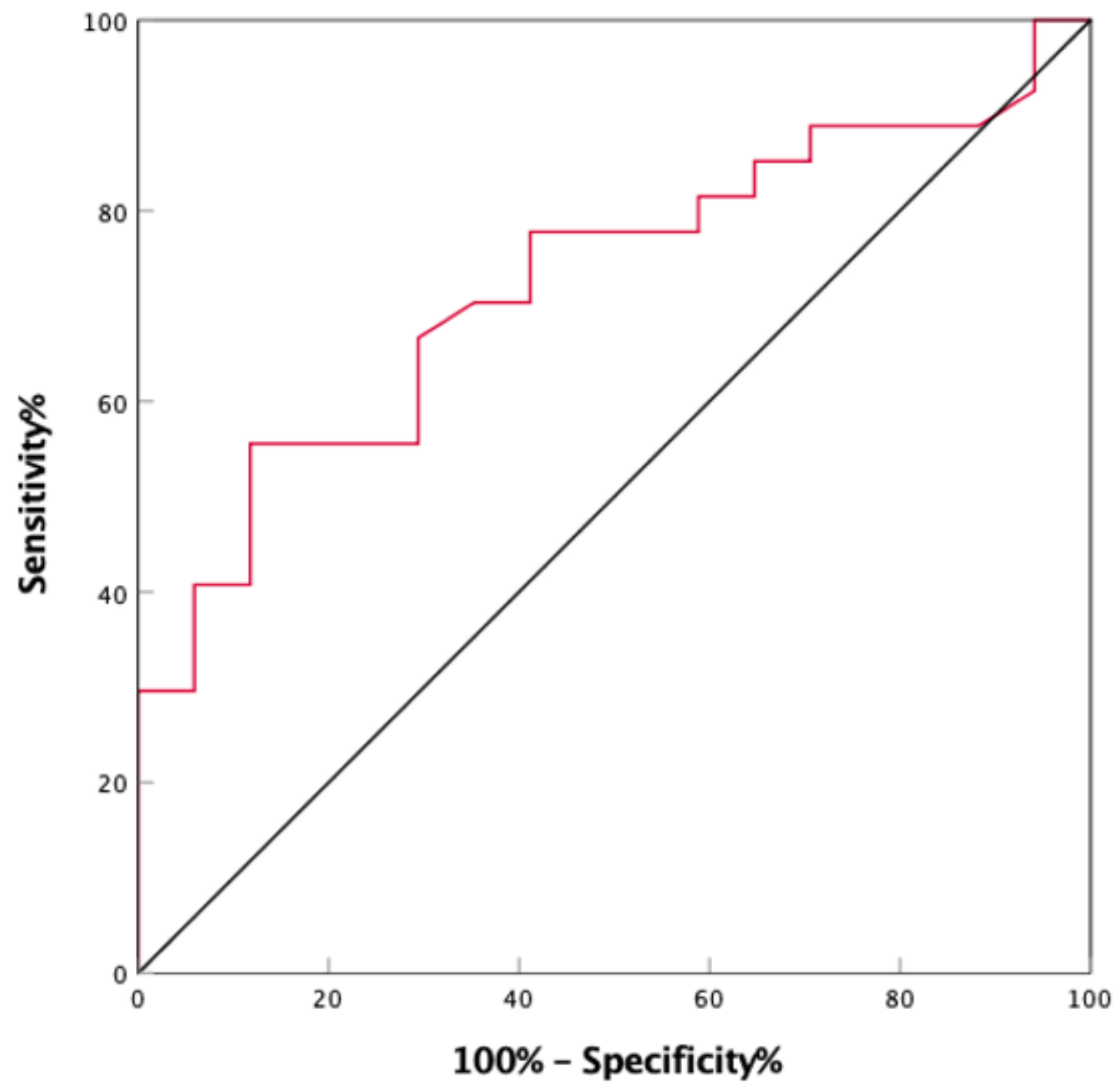

Figure 6

Predictive value of sputum cell CC16 mRNA relative expression level for COPD severity (FEV1\%pred) according to receiver operating characteristic $(\mathrm{ROC})$ curve analysis. Cutoff point $=1.09$; sensitivity $=55.6 \%$; specificity $=88.2 \%$; area under the ROC curve $(A U C)=0.725 ; 95 \% \mathrm{Cl}=0.577-0.874$. 